wir in Maснатscheк nur Rudimente, so * Das Gebiet der meridionalen Stromfurchen * (Band II) eine fast rein orographische Beschreibung.

Die zweite Stufe, die die orographische Erforschung roraussetzt, ist die geologisch-tektonische Betrachtung, die den Stil des Reliefs aus dem Innern, aus dem Bau, begründet. Diese Stufe kann sich schon auf ein Standardwerk der letzten Generation, auf ED. SuEss' Antlitz der Erde, stützen. In der übersichtlichen Ausbreitung der einschlägigen Literatur liegt der eine wichtige Akzent von Machatscheks Arbeit; der Geograph wird hier mit Nutzen Auskunft holen.

Der Hauptakzent von Machatschexs Werk liegt aber auf der morphotektonischen Beschreibung des Reliefs. Da bewundern wir die ausgedehnte Belesenheit des Autors und die kritisch sichtende Darstellung eines an sich oft recht abstrakten Stoffes. Hier gilt es, das Zusammenspiel von endogenen und exogenen Vorgängen, von Hebungen und Abtragungen, von Senkungen und Auffüllungen, zu erfassen und zeitlich einzuordnen. Es sind vor allem Einebnungsflächen, die eine mehr oder weniger sichere Datierung der einzelnen Phasen erlauben. Маснатsснек verschweigt nicht die Schwierigkeiten der Deutung, die sich aus unsern vielerorts noch mangelhaften Kenntnissen ergeben. Es zeigt sich auch, daß bei klẹnräumigen Studien die chronologische Bedeutung mancher zufälligen und vieldeutigen Kleinform überschätzt und ein kleinliches System errichtet wird, das sich kaum für das betreffende Gebiet, sicher aber nicht für Nachbargebiete eignet. MaChatscheks kritische Bemerkungen zeigen, daß die übertriebene Verfeinerung der Methode nicht nur in den Alpen zu ungehemmten Spekulationen führt $(\star$ Weitere Versuche, eine vielgliedrige Folge von Eintiefungsund Verebnungsphasen aufzustellen [25, 22 oder 19 Phasen] sind als unkritisch abzulehnen.*), sondern in allen bis zum Kleinraum durchforschten Gebieten. (Großbritannien : * Stockwerkflächen ... ohne daß bisher über ihre $Z$ ahl und auch über ihre Entstehungsweise ein endgültiges Urteil oder ihre Parallelisierung über große Käume möglich wäre .... Appalachen: ‘... Bemühungen um Aufstellung möglichst vieler Niveaus...*). Diese und viele andere Beispiele zeigen, daß die überspitzte Verfeinerung der Phasenchronologie manche spekulative Scheinblüte hervorgebracht hat.

Machatscheks Werk vermittelt einen weiten Blick auf das Gebiet der Erforschung des Reliefs, und wir freuen uns, da $\beta$ es, nachdem die erste Auflage durch Einwirkungen des Krieges zum größten Teil zerstört wurde, nun wieder als ein Markstein einer ganzen Forschergeneration in zweiter Auflage neu erstehen konnte. Es bietet aber, wie jedes Standardwerk, nicht nur einen Rück- und Überblick, sondern ermöglicht uns auch einen Ausblick mit weiten Perspektiven auf noch unerschlossene Forschungsgebiete des Reliefs der Erde.

\title{
DIE NEUEN OBERSTUFENATLANTEN DES GEOGRAPHISCHEN INSTITUTES ED. HÖLZEL IN WIEN
}

\section{EduARD ImHoF}

Seit 1951 erschienen in rascher Folge neue, durch das Geographische Institut Ed. Hölzel in Wien erstellte Oberstufenatlanten für Österreich, Frankreich und Deutschland.

Es sind dies:

1. der e Osterreichische Mittelschulatlas , bearbeitet von Hass StanaR und herausgegeben vom Verlag Ed. Hölzel, Wien 1951 (eine Neubearbeitung des Kozenn-Atlasses).

2. der - Nouvel Atlas Général , bearbeitet von Pierre Serryx, René Blasselle und Marc Bonnet, herausgegeben von Bordas, Paris 1953.

3. der -Atlas zur Erdkunde s, bearbeitet von Hermans LautensaCH, herausgegeben von der Keyserschen Verlagsbuchhandlung, Heidelberg, erste Auflage 1954, zweite Auflage 1955.

Diese drei Atlanten stimmen sowohl im Umfang (146-148 Kartentafeln), Buchformat $(22 \times 30,5 \mathrm{~cm})$, wie auch im graphischen Charakter der Karten miteinander überein. Und nicht nur das, mehr als die Hälfte der Blätter ist, abgesehen von Korrekturen und Nachträgen und abgesehen von der ins Französische übersetzten Beschriftung des «Bordas》, in diesen Atlanten identisch. Dies ergibt sich aus ihrem gemeinsamen Ursprung, denn nicht nur die Reproduktion der Karten, sondern auch ein GroBteil ihrer inhaltlichen Bearbeitung und Zeichnung wurde in Wien durch das Geographische Institut Ed. Hölzel besorgt. Der Grundstock der Karten erschien erstmals im Österreichischen Mittelschulatlas 1951. Etwa 5o Kartentafeln zur Landeskunde Österreichs wurden jedoch für die französische Ausgabe durch Karten französischer Gebiete, für die deutsche Ausgabe durch solche von Deutschland ersetzt. Eine entsprechende Umformung für einen demnächst erscheinenden englischen Atlas ist in Arbeit.

Es wäre nicht richtig, solche Export-Erfolge der Wiener Schulkartographie nur der Geschäftstüchtigkeit des Hölzel-Verlages zuschreiben zu wollen. Inhalt und Form der Atlanten werben für sich. Die Buchformate sind kleiner und handlicher als diejenigen der meisten bisherigen Oberstufenatlanten, auch kleiner als das Format unseres Schweizerischen Mittelschulatlasses. Dies erleichtert ihren Absatz, wird aber andererseits durch Mängel erkauft, auf die wir unten zurückkommen werden. Was diese neuen Wiener Atlanten besonders anziehend 
macht, ist ihre saubere, freundliche, farbig aufgehellte graphische Haltung, ist ferner ihr Reichtum an Karten und deren geschickte Auswahl. An Kartenreichtum übertreffen sie die meisten ausländischen Oberstufenatlanten, nicht aber den Schweizerischen Mittelschulatlas.

Der inhaltliche Aufbau des österreichischen sowohl wie des deutschen Atlasses entspricht bewährten didaktischen Ansichten. Daraus ergeben sich folgende Kartengruppen: Einführung in die Karte und Beispiele staatlicher Karten, Landeskunde des eigenen Staatsgebietes, ausländische europäische Staaten und Gebiete, Europa als Ganzes, übrige Erdteile, Ozeane, Polargebiete, Erde als Ganzes, Himmel. Diese kleineren und größeren Räume kommen jeweils in Übersichtskarten und in der Regel auch in thematischen (angewandten) Karten und Detailkarten wichtiger oder besonders interessanter Teilgebiete zur Darstellung. Der französische Atlas verzichtet auf Karteneinführung und Himmelskunde. Er stellt die Erdübersichten, die Polar- und Ozeankarten und diejenigen von Europa an den Anfang. Da ein Atlas jedoch nicht wie ein Lehrbuch von vorn nach hinten durchgearbeitet werden muß, ist diese Abweichung von der didaktischen Norm praktisch von geringer Bedeutung.

Die Karten des eigenen Landes (im Bordas-Atlas diejenigen des französischen Mutterlandes und der überseeischen Gebiete) nehmen in diesen neuen Atlanten mit 48-53 Seiten je etwa ein Drittel aller Kartentafeln ein. Die Atlanten sind somit stark auf das jeweilige «Verbrauchergebiet» abgestimmt. Gerade darum sind sie jedem Geographen als Spezialatlanten von Österreich, Deutschland und Frankreich willkommen.

Der Schweizerische Mittelschulatlas kommt mit 27 Kartentafeln des eigenen Landes aus, ohne deshalb hierüber weniger zu bieten. Dies ist einer der Vorteile des Kleinstaates. Bei ungefähr gleich großer Total-Seitenzahl steht daher im Schweizer Atlas mehr Raum für ausländische Gebiete zur Verfügung. Der Schweizer Atlas gibt u. a. für jedes europäische Teilgebiet neben einer Haupt- oder Übersichtskarte einige thematische und regionale Nebenkarten, was in den neuen Hölzel-Atlanten nicht oder nur in beschränktem Maße der Fall ist. Die außereuropäische Welt beansprucht in den Hölzel-Atlanten etwa gleich viel Raum, wie im Schweizer Atlas. In der Zuteilung des Stoffes aber bestehen interessante Unterschiede. Die Hölzel-Atlanten bringen die fremden Erdteile im Maßstab 1:20000000, hierbei Asien und Afrika freilich in Teilkarten zerlegt. Die Erdteil-Karten des Schweizer Atlasses aber besitzen, abgesehen von Europa, nur den Maßstab 1:30000 ooo. Dafür aber sind hier für alle wichtigen Räume (Orient, Vorder- und Hinterindien, China und Japan, Sunda-Inseln, zentrales Rußland, Nordafrika, Vereinigte Staaten, Mittelamerika und Antillen, Teile von Südamerika und Australien) Karten in wesentlich größeren Maßstäben beigefügt.

Alle drei Hölzel-Atlanten leiden an einer zum Teil wohl wèit zurückliegenden Fehldisposition. Man wollte - um ein schweizerisches Wort zu gebrauchen - den «Fünfer und das Weggli». Man wollte möglichst kleine Buch- und Kartenformate und gleichzeitig möglichst große Gebiete und Maßstäbe. Dies führte zu Rahmen- oder Randdurchbrechungen und unschönen Karten-Ineinanderschachtelungen in einem Ausmaß, das der Schönheit der Werke Abbruch tut. Jeder Atlas-Ersteller sieht sich da und dort zu Auskropfungen der Karten genötigt. Hier aber dürfte das ästhetisch erträgliche $\mathrm{Ma} B$ überschritten sein. Über die Hälfte aller Karten sprengen ihre Rahmen. Unter solchen Umständen wäre eine kleine Vergrößerung der Buchund Kartenformate das kleinere Übel gewesen. Besonders schlimm wirkt sich die $Z_{\text {wangsjacke }}$ des Formates im Lautensach-Atlas aus, wo deutsche Räume nach allen Seiten hin die Ränder überfließen. Überall platzen die Nähte, so wie wenn ein wohlbeleibter Herr sich in eine Knabenbadehose zwängt. Ein krasses Beispiel hierfür ist die Karte «Schlesien und Nordböhmen» auf Seite 21 der zweiten Auflage. Um den Ostzipfel von Schlesien zu retten, mußte er trotz Randüberlappungen amputiert und in die polnische Kartenecke gesetzt werden.

Der Eindruck eines jeden Atlasses wird wesentlich mitbedingt durch Art und Qualität der Geländedarstellung. In dieser Hinsicht verdienen die neuen Hölzel-Atlanten alles Lob. Harmonische, konsequent abgestufte und durch feine Höhenlinien konturierte Höhenfarbtöne verbinden sich mit einer sorgfältig durchgearbeiteten Geländeschummerung. In ihrer farbigen und plastischen Haltung sind diese Karten denjenigen der früheren Slanar-Atlanten des Kartographischen Institutes in Wien ähnlich. Neu ist gegenüber jenen die Konturierung der Farbstufen und der Ersatz der Schraffen durch Schummer. Es müssen jedoch hierzu einige Klarstellungen angebracht werden, die nicht die Karten betreffen, wohl aber was darüber geschrieben worden ist. Erstens ist die Darstellungsart (Höhenfarben mit Höhenlinienkonturen und Schummerung) keineswegs neu. Sie gelangte schon oft zur Anwendung. $Z$ weitens ist es noch längst keine ausgemachte Sache, da $\beta$ eine Schummerung in vorliegender Form unbedingt besser sei als entsprechende Schraffen. Sie ist wohl billiger und leichter zu erstellen. Dies ist ein Hauptgrund ihres Siegeszuges in der modernen Kartographie. Mit beiden graphischen Elementen, mit der Schraffe und mit der Schummerung, bezweckt man dasselbe, man will damit bestimmte, miniaturhaft feingliedrige Verdunkelungen erzeugen. Nirgends aber in der Graphik, auch nicht in der Kartengraphik, steht es fest, da $\beta$ Tonabstufungen durch Schummer solchen durch Schraffuren stets und eindeutig überlegen seien. Oft ist das Gegenteil der Fall. Die Schraffe akzentuiert schärfer und prägnanter, sie ist solider, drucktechnisch sicherer. Infolge ihrer Gitterstruktur verschleiert sie die Höhenfarbtöne weniger. Sie gibt nicht nur Toneffekte, vielmehr zeigt sie 
durch die Strichrichtungen auch die Gefällsrichtungen. Man halte daher in solchen Dingen mit voreiligem Urteil zurück. Der Schummer freilich läßt sich freier und weicher modellieren und den tatsächlichen Geländeformen besser anpassen. Er ist weniger steif als die Schraffe. Durch genaueste Imitation des Licht- und Schattenspiels, wie es bei flachem Lichteinfall auf topographischen Flächen entsteht, führt er zu Abbildungen, die in hohem Maße unwillkürlich plastisch erscheinen. Solche Darstellungen nennt man Reliefkarten. Dieser Schritt zur schattenplastischen Impression aber ist in den Länderkarten der neuen Hölzel-Atlanten ebensowenig gemacht, wie in den entsprechenden Schraffenkarten des Schweizer Mittelschulatlasses. Die Darstellungen hier wie dort sind immer noch traditionsgebunden und in ihren graphischen Effekten grundsätzlich gleichartig. Mit diesen Ausführungen sei nicht die Geländedarstellung der Hölzel-Atlanten beanstandet, jedoch eine Angabe von Prof. LAUTensach (im Vorwort seines Atlasses) berichtigt, es handle sich hier um «Reliefkarten». In der Schweiz wenigstens, wo die Reliefkarte entwickelt worden ist, versteht man darunter nicht jede geschummerte Geländedarstellung, sondern nur eine solche, die konsequent schattenplastisch gegliedert ist. Schummer und Schatten sind visuell verschiedene Dinge. Reliefkarten sind u. a. die regionalen Karten im Anfangsteil des Schweizer Mittelschulatlasses, ebenso die sog. Wenschowkarten. Die Karten auf den Seiten 6-9 des neuen Österreichischen Mittelschulatlasses (Ausgabe 1951) sind verheißungsvolle Versuche dieser Art. Auch die Deutschland-Ausgabe enthält zwei solche Proben, nämlich die dem österreichischen Atlas entnommene Großglocknerkarte und RICHARD FINSTERWALDERS instruktive Nanga Parbat-Karte.

$\mathrm{Zu}$ den ansprechendsten und nützlichsten Karten der neuen Hölzel-Atlanten zählen die Bodennutzungskarten der Erdteile. In der französischen, resp. deutschen Ausgabe wird ihr Inhalt treffender mit «Couverture végétale», resp. «Bodenbedeckung» umschrieben. Sie weisen in den drei Atlanten beträchtliche Unterschiede auf. Sowohl die französischen Bearbeiter, wie besonders H. LAUTENSACH scheuten keine Mühe, diese Karten wesentlich zu verbessern. Inhaltliche und graphische Konzeption aber gehen auch hier auf die österreichische Bearbeitung durch Hans Slanar und seine Mitarbeiter zurück. Im Gegensatz zum Schweizer Mittelschulatlas, wo Vegetation und Bodenbewirtschaftung getrennt zur Darstellung gelangen, werden hier diese Dinge vereint gezeigt. Die Farbhaltung der Karten erinnert in großen Zügen an die äußeren landschaftlichen Aspekte, teilweise auch an die Farben bekannter Produkte. Es ist interessant, solche bis zu einem gewissen Grade naturalistische Darstellungsweise zu vergleichen mit derjenigen des Schweizer Atlasses. Der letztere gibt die Vegetationsgebiete in abstrakteren, damit aber auch besser unterscheidbaren klimazonalen Farbabstufungen und sucht intensive, resp. extensive (üppige und magere) Vegetations- und Wirtschaftsgebiete durch satte, resp. blasse Farben zu charakterisieren. Ähnlich wie die Erdteilkarten der Bodenbedeckung sind im österreichischen und französischen Atlas auch die Vegetations-Erdübersichtskarten aufgebaut. Die entsprechende Karte des Lautensach-Atlasses erscheint weniger glücklich. Das natürliche Pflanzenkleid der Erde (Seite 133) wird hier teils durch naturalistische, teils wärmezonale, teils sonstige und oft möglichst kontrastierende Farben zum Ausdruck gebracht. Die Karte ist zwar bildhaft schön, jedoch verwirrend. Gegensätzliche Dinge (Hochgebirgswüsten und tropische Urwälder) erscheinen in ähnlichem Grau und Grauviolett. Verwandte Erscheinungen aber, wie Savannen und Dornbuschsteppen, besitzen die kontrastierenden (komplementären) Farben Rot und Grün. Subtropische, immerfeuchte Urwälder, somit ausgesprochene Intensivgebiete (z. B. in China und im Süden der USA) sind Blaßgrau gefärbt, ähnlich wie Tundren und subpolare Wiesensteppen.

Sehr klar, vielleicht etwas stark vereinfacht sind auch die Wirtschaftskarten der Erdteile. Auch hier verraten die Unterschiede der drei Ausgaben die ergänzende und berichtigende Hand des einzelnen Überarbeiters. Das Bemühen um Einfachheit und Klarheit ist zweifellos zu begrüßen. Manchmal tritt dann aber durch allzu markante graphische Sprache auch eine allzu markante Ungereimtheit zu Tage. In der Wirtschaftskarte von Europa, auf Seite 80 der Deutschland-Ausgabe, umschließen kräftige, rote Stränge das Fleisch- und Milchwirtschaftsgebiet. Das rosagefärbte, baltische Milchwirtschaftsgebiet (Tilsit usw.), ebenso das mit «Milchvieh» angeschriebene skandinavische Gebiet aber liegen in der Karte außerhalb dieses Grenzbandes. Auch die Südgrenze des Milchviehs ist irreführend zusammengefaßt. Sie müßte den Kaukasus und das armenische Hochland umschließen, wo die Rinderzucht (Yoghurt usw.) weit verbreitet ist. Wozu also derart markant kartieren, was gar nicht so einfach abgegrenzt werden kann?

In unmittelbarer Nachbarschaft dieser großen Wirtschaftskarten stehen Industriekärtchen der Erdteile in 1:100000000, für Europa in 1:50000 000. Ebenfalls in diesem letztgenannten Maßstab sind für Europa Geologie, Bodentypen, Wirtschaftsformen, Völker usw. dargestellt. Ich möchte den Wert solch miniaturhaft kleiner Kärtchen, in welchen West- und Mitteleuropa die Flächengröße einer normalen Briefmarke besitzt, bezweifeln. Jede Stichprobe erweist deren Versagen. Geologie (im Gegenatz zu den großen tektonischen Einheiten) läßt sich bei solcher Zusammenfassung nicht sinnvoll darstellen. An Hand der Industriekarten sollten u. a. Standortfragen erörtert werden. Dies ist bei solcher Mikrokartierung unmöglich. Die Bezeichnung «Völker» ist nicht richtig. Es handelt sich hier um Sprachgruppen. Was aber hilft eine solche 
kleine Karte, die es z. B. nicht mehr erlaubt, portugiesische, spanische, französische und italienische Sprachen gegeneinander abzugrenzen und in den Alpen die rätoromanischen Gebiete einzutragen?

Auf die positive Seite wiederum ist in jedem der drei Hölzelatlanten die Darstellung der wahren, nicht reduzierten Januar- und Juli-Isothermen des betreffenden Landes (Österreich, Frankreich, Deutschland) zu buchen. Der Bordas-Atlas im besonderen gibt für Frankreich in höchst instruktiver Weise sowohl die wahren, wie auch die reduzierten Isothermen. Im weiteren geben alle drei Atlanten Isothermenkarten für Europa, für Asien und für die Erde. Für die übrigen Erdteile fehlen sie. In der Europakarte sind die Isothermen reduziert, in der Asienkarte aber nicht! Raum- und Quellenmangel können solche Inkonsequenzen kaum genügend rechtfertigen. Im österreichischen und im französischen Atlas sind in der entsprechenden Weltkarte, somit in ein und derselben Karte für dàs asiatische Gebiet nicht reduzierte, für die übrigen Erdteile aber reduzierte Isothermen eingetragen, ohne überhaupt anzugeben, ob es sich um diese oder jene handelt. LAUTENSACH hat solches Wirrwarr in seiner Ausgabe beseitigt. Auch viele andere Dinge hat er bereinigt. Unter anderem ist sein Atlas der einzige der drei Hölzel-Atlanten, der konsequent für jeden Erdteil eine Niederschlagskarte enthält. In der österreichischen und französischen Ausgabe fehlen sie seltsamerweise für Asien. Er hat Inhalt und Legende der geologischen Erdkarte ergänzt und verbessert, und er hat darin besser zu unterscheidende Farben eingeführt. In den beiden anderen Atlanten sind zwar diese Karten graphisch sehr schön, vielleicht schöner als bei LAUTENSACH, doch lassen sich ihre Farben zum Teil kaum voneinander unterscheiden.

So gäbe es noch viel zu sagen, viel Positives und auch einiges Negative. Dies aber würde den Rahmen unserer Besprechung sprengen. Wie jedes solche Werk enthalten auch die HölzelAtlanten lokale Einzelfehler. Bei Erstausgaben neuer Atlanten sind sie Folgen mangelhafter Quellen oder redaktioneller und zeichnischer Versehen. Sie werden nach Möglichkeit von Auflage zu Auflage vermindert. Bei späteren Auflagen aber treten infolge Veraltens und ungenügender Erfassung von Veränderungen oft neue Fehler hinzu. Jeder Atlasbearbeiter steuert durch tausend Klippen. Seine Arbeit gleicht dem Kampf des Herakles gegen die Hydra. Einzelfehlermeldungen sind jedem Kartenredaktor willkommen, doch gehören sie nicht in eine Buchbesprechung, denn es handelt sich hierbei meist um Zufallstreffer, die dem Leser wenig nützen. Rezensenten, die nur solches zu melden wissen, wollen ja damit lediglich ihr eigenes Flämmchen leuchten lassen. In einer Atlasbesprechung sind nur methodische und grundsätzliche Dinge angebracht. In diesem Sinne sei noch kurz auf ein letztes, die drei Atlanten gemeinsam berührendes Thema eingegangen, auf die Kartennetze und deren Bezeichnungen.

Die Wahl der Netze (Projektionen) ist in den drei Hölzel-Atlanten durchwegs glücklich, sie entspricht heutigen Erfahrungen und Möglichkeiten. Zweckmäßig war besonders auch die Wahl von ECKERTs flächentreuer Sinuslinienprojektion für zahlreiche Erdkarten. Diese Netze präsentieren sich sehr gut, sowohl in den ganzseitigen Karten, wie auch da, wo drei Karten auf einer Seite vereinigt sind. Das Merkatornetz verschwindet infolge seiner starken Flächenverzerrungen mehr und mehr aus den Schulatlanten. Vom Standpunkt der Netzlehre aus wäre zu wünschen, $d a B$ es wenigstens in einem Erdkartenbeispiel, etwa auf einer Seite mit klimatologischen Karten, zur Anwendung gelangt wäre. $Z$ u begrüßen sind die bei vielen Karten angebrachten Hinweise auf die Art der Netze. Im Gegensatz zum Lautensach-Atlas, der in dieser Hinsicht einwandfrei ist, erweisen sich einige der Netzhinweise des österreichischen Atlasses als veraltet, als unrichtig oder unvollständig. Wer spricht heute noch von homalographischer Projektion (Seite 142-144) für MolLweides flächentreue, unechte Zylinderprojektion? Die beiden Polarkarten sind normale (oder polare) Azimutalprojektionen mit wahren Abständen vom Kartenmittelpunkt $(\mathrm{Pol})$ aus. Der österreichische Atlas bezeichnet sie fälschlicherweise als Kegelprojektionen.

Die zur Hauptsache dem einstigen Sydow-Wagner Schulatlas entstammenden Darstellungen der Gradnetzentwürfe bei Blatt 1 der deutschen Ausgabe halte ich für entbehrlich. Sie reicht selbst für eine sehr elementare Einführung in die Netzlehre nicht aus, enthält mehrere Netze, die im Atlas nicht vorkommen, und läßt Netze vermissen, die zur Anwendung gelangten.

Nach diesen Gesamtbeurteilungen der drei Atlanten sei jeder einzelne derselben nochmals ganz kurz betrachtet.

Zunächst der «Österreichische Mittelschulatlas》. Methodischer und redaktioneller Betreuer war zur Hauptsache der im Sommer 1955 verstorbene ausgezeichnete Wiener Schulgeograph Prof Dr. Hans Slanar. Er schuf, zusammen mit Dr. Hugo Eckelt, Oskar Neoral, Wilhelm VELC und weiteren Mitarbeitern der Kartographischen Anstalt Hölzel das Basiswerk aller drei Atlanten.

Bemerkenswert am österreichischen Atlas sind unter anderem besonders die schönen Karten österreichischer Landschaften, Kalkhochgebirge (Rax), Mittelgebirge (Wachau), vergletschertes Hochgebirge (Großglockner), alle im Maßstab 1:50 ooo. Einige weitere Landschaftskarten leiden an zu heftig-grünem Waldton. Starkes Interesse bieten auch die Karten der Stadttypen, der Dorf- und Flurformen und all die zahlreichen thematischen Karten von Öster- 
reich, ferner die Karten des Donaudeltas, der Finnischen Seenplatte, der Wolga bei Stalingrad usw. Die Pläne südamerikanischer Plantagen und Farmen sind zwar ebenfalls lehrreich, beanspruchen aber infolge ihrer unnötig großen Maßstäbe zu viel Raum.

Der «Nouvel Atlas Général», herausgegeben von Bordas, Paris. Seine redaktionellen Betreuer wurden oben genannt. Bemerkenswert sind hier die zahlreichen, ausgezeichneten Karten von Frankreich und der überseeischen, französischen Gebiete. Die Themakarten dieser Gruppe stützen sich auf neuestes Quellenmaterial. Methodisch sehr geschickt sind die Zusammenstellungen von je fünf Karten eines jeden Landesteiles (Geländekarte, Geologie, Pflanzendecke, Niederschläge, Volksdichte), obschon einige dieser Karten Wiederholungen der betreffenden Landes-Gesamtkarten sind. Bemerkenswert sind auch die zahlreichen Karten 1:200 000 französischer Städte, mit Hervorhebung der Industrie- und Verkehrsanlagen.

Der für die Schulen Deutschlands bestimmte «Atlas zur Erdkunde des Kayser Verlages wurde von HeRmann LAUTENSACH betreut. Wie der Wiener Atlas enthält er einleitend einige Gegenüberstellungen von Luftbildern (Photos) und Karten. Im Stuttgarter Beispiel der ersten Auflage 1954 waren die Bild- und Karteninhalte nur mit Mühe zu identifizieren. Das ausgewechselte Luftbild der Ausgabe 1955 erfüllt seinen Zweck nicht viel besser. Blickfeldbegrenzungen der Photos sollten bei solchen Gegenüberstellungen in die Karten eingetragen werden. Damit würde das Vergleichen sehr erleichtert. Auch im Keyser-Lautensach-Atlas fesselt uns besonders der Deutschland gewidmete Anfangsteil. Wir finden hier u. a. eine gute, doppelseitige Karte des Rheinisch-westfälischen-Industriegebietes in 1:200 000 , lehrreiche Gegenüberstellungen von Relief und Geologie des Thüringer Waldes und eines Teiles der Schwäbischen Alb, ferner eine große Karte des geologischen Baues von Mittel- und Westeuropa. Ihre vorzügliche inhaltliche Gliederung und der schöne klare Farbaufbau werden jedoch beeinträchtigt durch eine gewisse Manierhaftigkeit der zeichnerischen Generalisierung. Viele Farbflächen sind unnötig gerundet, sehen aus wie bunte Wassertropfen oder gar wie gefärbte Ostereier. Sie weichen allzusehr ab vom Formencharakter geologischer Aufschlüsse und lassen in den Westalpen und an manch anderen Orten die natürlichen Zusammenhänge nur schwer erkennen. Dieselbe lineare Manierhaftigkeit zeigt freilich auch die geologische Frankreichkarte des schon vorher erschienenen Bordas-Atlasses.

Die zweite Auflage des Keyser-Lautensach-Atlasses, diejenige von 1955, weicht im Deutschlandteil wesentlich von derjenigen des Jahres 1954 ab. Da Deutschland heute noch keinen Friedensvertrag besitzt, bestehen gewisse politische Zustände im Osten des Landes wohl de facto, nicht aber de jure. Diesem Umstand wurde in der ersten Auflage zu wenig Rechnung getragen. Der zweiten Auflage wurden dann auf Wunsch deutscher Kreise eine politische Karte des Vorkriegsdeutschland und Karten der seither besetzten ostdeutschen Gebiete in größeren Maßstäben beigefügt.

Den Bearbeitern des «Nouvel Atlas Général» und des «Atlas zur Erdkunde» kommt zweifellos das Verdienst zu, für Frankreich, resp. für Deutschland erstmals in der Nachkriegszeit einen modernen und kartentechnisch guten Oberstufenatlas herausgebracht $\mathrm{zu}$ haben. Leider aber verursachen die Flaggen, unter denen dies geschehen ist, ein gewisses Unbehagen.

Wir stellen nochmals fest: Mehr als die Hälfte der Karten, sowohl des französischen, wie des deutschen Atlasses sind, abgesehen von Korrekturen, Nachträgen und sprachlichen Anpassungen der Nomenklatur, identisch mit denjenigen der früher erschienenen österreichischen Ausgabe. Einige weitere Karten, vor allem solche der Bodenbedeckung und Wirtschaft, wurden zwar überarbeitet und verbessert, sind aber im übrigen ebenfalls dem österreichischen Atlas entnommen. Je etwa ein Drittel (der Frankreich-, resp. Deutschlandteil) sind neu disponiert und neu bearbeitet, jedoch kartentechnisch ebenfalls durch die Anstalt Hölzel in Wien erstellt (nicht nur gedruckt) worden. Rechtlich ist dagegen nichts einzuwenden, da sich die beteiligten Verleger hierüber verständigt hatten. Der französische Atlas nennt auf dem Titelblatt lediglich die Autorennamen SERRYN, BLASSELle und BoNNET. Im Vorworttext lesen wir die mehr als seltsame Bemerkung «Les auteurs ont utilisé également (d.h. in derselben Weise, wie andere Quellen) un certain nombre de cartes publiées par le Professeur SlaNar de l'Institut Géographique de Vienne». Auf dem hintersten Leerblatt findet sich sehr klein der Hinweis, der Atlas sei durch Hölzel in Wien «gedruckt» worden. Kartenherstellen und bloßes Kartendrucken sind aber ebensowenig dasselbe wie Bücherschreiben und Bücherdrucken! Ähnliches ist leider in der deutschen Ausgabe festzustellen. Im Titelblatt wird Lautensach als «Bearbeiter» vorgestellt. Das Vorwort der zweiten Auflage enthält als einzige Hinweise auf den Wiener Ursprung folgende Stellen:

«Diese Mcthoden der Reliefdarstellung sind auch die des Österreichischen Mittelschulatlasses, mit dem der vorliegende in den Projektionen, Maßstäben und Schnitten einer ganzen Reihe von außerdeutschen Karten übereinstimmt». In der Ausgabe des Jahres 1954 fehlt auch dieser Hinweis. Schließlich wird dann zahlreichen Persönlichkeiten und Instituten und dabei auch den «Mitarbeitern im Geographischen Institut Hölzel - Wien》für ihre Hilfe gedankt. Auch in diesem Atlas erscheint Hölzel nur als «Drucker». Der Name des ursprünglichen Hauptbearbeiters, des indessen verstorbenen Prof. Dr. Hans SLANAR fehlt. Im Vorwort der er- 
sten Ausgabe des «Lautensach-Atlasses》 fand sich immerhin die Feststellung, daß sowohl « Zeichnung》 und «Druck» durch das Hölzel-Institut erfolgt seien.

Bearbeiter einer Karte ist nur, wer ihren Inhalt und ihre Form bis ins Kleinste bestimmt oder festlegt. Hier aber sind Karten anderer Bearbeiter übernommen und in manchen Fällen revidiert worden, ohne dies richtig zu sagen. «Projektion, Maßstab, Blattschnitt und Methoden» machen eine Karte noch längst nicht aus. Der genannte Hinweis im Lautensach-Atlas erweckt beim unbefangenen Leser den Eindruck, Inhalt und Form, somit die Bearbeitung der betreffenden Karten, seien ihm zuzuschreiben. Ein Verleger darf einem Atlas seinen Namen geben. Dies ist weitherum üblich. Jedermann weiß, daß er den Atlas nicht selbst bearbeitet hat. Ein Wissenschafter aber soll in solchen Dingen korrekter sein.

Diese Feststellungen mußten gemacht werden. Ich bedaure es aufs tiefste, daß ich hierzu genötigt war.

\section{GESELLSCHAFTSTÄTIGKEIT - ACTIVITÉ DES SOCIÉTÉS}

Vortragstätigkeit im Wintersemester 1955/56. Basel. 28. Oktober. PD. Dr. H. Carol, Zürich: Reisen und Forschungen in Ostafrika; 11. November. Dr. U. RAHM, Basel: Streifzüge durch die Elfenbeinküste; 25. November. Dr. H. Dietschy, Basel: Reise zu den Karaja-Indianern Zentralbrasiliens; 9. Dezember. Prof. Dr. N. Creutzburg, Freiburg i. B.: Florenz und die Toskana; 16. Dezember. Dr. J. Høssi, Männedorf: Vom Heidenhüttli zum modernen Alpgebäude; 13. Januar. Prof. A. Glory, Straßburg: La Préhistoire Maroccaine; 27. Januar. Dr. E. Schlager, Basel: Bali; 10. Februar. Frl. Dr. E. Leuzinger, Zürich: Bei den Afo im nigerischen Busch; 24. Februar. Dr. H. LEHmann, Paris: Guatemala ; 9. März. Prof. Dr. H. GeEtz, Freiburg i. B.: Indische Kunst und Religion. Bern. 16. Oktober. R. Studer, Bern: Das unbekannte Bern (Führung); 21. Oktober. Prof. Dr. A. Heim, Zürich: Die Schweizerische Virunga-Expedition in Zentralafrika 1954/55; 5. November. R. Studer, Bern: Das alte Bern; 11. November. R. Gard, Bern: Unter Färbern und Webern in Nord-Kamerun; 18. November. PD. Dr. W. Staub, Bern: Die Tropen als Wirtschaftsraum : 25. November. O. Jenny, Oberdorf: Pyrenäen und Baskenland; 16. Dezember. H. Sturzenegger, Bern: Sardinien. St. Gallen. 27. Oktober. E. Reber, St. Gallen: Reisebilder aus Persien (1954); 15. November. H. R.-G. JÆckel, Wiesbaden: Das heutige Grönland; 6. Dezember. Prof. Dr. E. EgLI, Zürich: Der Berg Ararat (Expedition 1954); 17. Januar: Prof. Dr. H. Louls, München: Die neue Türkei ; 7. Februar. Prof. Dr. G. Pfeifer, Heidelberg: Ost-Brasilien zwischen Rio und Bahia (1955); 28. Februar. Prof. Dr. H. Kinzl, Innsbruck: Peru von heute (Anden-Expedition 1954); 13. März. Prof. Dr. O. Widmer, St. Gallen: Ferner Osten - Schicksalsraum der Welt. Zïrich. 2. November. Prof. Dr. A. Heim, Zürich: Bilderbericht der schweizerischen Virunga-Expedition in Zentralafrika 1954/55 ; 16. November : Prof. Dr. H. Boesch, Zürich : Chicago ; 30. November. Frl. Dr. E. LeuZinger, Zürich : Forschungen bei den Afo-Negern im nigerischen Busch; 14. Dezember. Prof. Dr. J. SchmirHÜSEN, Karlsruhe: Chilenische Landschaften; 11. Januar. Prof. Dr. E. Imнof, Zürich: Reliefkarten kleiner Maßstäbe (Fachsitzung); 18. Januar. Prof. Dr. H. Louis, München : Die neue Türkei; 25. Januar. Prof. Dr. E. Schmid, Zürich : Übersicht über die Vegetation der Erde (Fachsitzung); 8. Februar. Prof. Dr. G. Pfeifer, Heidelberg: Zwischen Rio und Bahia. Reisen und Forschungen im östlichen Brasilien; 22. Februar. Prof. Dr. F. GyGax, Bern: Hydrologisch-morphologische Beobachtungen in den Maggiatälern (Fachsitzung); 29. Februar. Prof. Dr. H. KInZL, Innsbruck: Peru von heute; 7. März. Prof. Dr. H. Goetz, Freiburg i. B.: Indien: Die indische Plastik und ihre kulturellen Grundlagen. Ende April: PD. Dr. H. Carol, Zürich: Ostafrika (Generalversammlung).

\section{REZENSIONEN - COMPTES - RENDU CRITIQUES}

Huber, H.: Was der Schweizerbiüger von seinem Vaterlande wissen soll. Ed. Rascher, Zurich 1955, 106 pages.

C'est sûrement en constatant, dans les examens des recrues, le manque de notions des jeunes Suisses sur l'aspect géographique et l'histoire de leur pays, et encore plus sur les institutions politiques de celui-ci, que l'auteur s'est décidé à publier cette petite brochure d'une centaine de pages, qui est un résumé succint mais très clair de la géographie, de l'histoire et des institutions politiques de notre pays. L'auteur met naturellement l'accent sur les particularités de notre forme de gouvernement et passe en revue toutes les notions que tout citoyen digne de ce nom doit connaitre. Cette brochure peut être vivement conseillée à tous les futurs citoyens, particulièrement aux élèves des écoles secondaires, tant que ces établissements n'ont pas introduit une solide instruction civique dans leurs programmes.

C. AUBERT

Institut fédéral pour l'étude de la neige et des avalanches. Schnee und Lawinen in den Schweizer Alpen, Winter $1952 / 53$. Ed. Buchdruckerei Davos S.A., Davos-Platz 1954, 120 pages, 57 figures.

Ce rapport est un compte-rendu de cet institut durant l'hiver 1952/53 qui fut très intéressant pour l'étude des avalanches, car il fut riche en neige. Après un résumé de l'évolution du temps et de la couche de neige durant les mois d'hiver, le rapport nous donne un bref aperçu de la structure du réseau d'observation et de prédiction des 